September $\quad 28, \quad 2018 \quad 13: 45$ WSPC/INSTRUCTION FILE proc magno machado stars2011

International Journal of Modern Physics E

(C) World Scientific Publishing Company

\title{
GEOMETRIC SCALING IN ULTRAHIGH ENERGY NEUTRINOS AND NONLINEAR PERTURBATIVE QCD
}

\author{
M. V. T. MACHADO \\ Instituto de Fisica, Universidade Federal do Rio Grande do Sul \\ Av. Bento Gonçalves 9500, CEP 91501-970. Porto Alegre, RS, Brazil \\ magnus@if.ufrgs.br \\ Received (received date) \\ Revised (revised date)
}

\begin{abstract}
The ultrahigh energy neutrino cross section is a crucial ingredient in the calculation of the event rate in high energy neutrino telescopes. Currently there are several approaches which predict different behaviors for its magnitude for ultrahigh energies. In this contribution is presented a summary of current predictions based on the non-linear QCD evolution equations, the so-called perturbative saturation physics. In particular, predictions are shown based on the parton saturation approaches and the consequences of geometric scaling property at high energies are discussed. The scaling property allows an analytical computation of the neutrino scattering on nucleon/nucleus at high energies, providing a theoretical parameterization.
\end{abstract}

\section{Introduction}

The investigation of ultrahigh energy (UHE) cosmic neutrinos provides an opportunity for study particle physics beyond the reach of the LHC 11. As an example, nowadays the Pierre Auger Observatory is sensitive to neutrinos of energy $\geq 10^{8}$ $\mathrm{GeV}^{2}$. A crucial ingredient in the calculation of attenuation of neutrinos traversing the Earth and the event rate in high energy neutrino telescopes is the high energy neutrino-nucleon cross section, which provides a probe of Quantum Chromodynamics (QCD) in the kinematic region of very small values of Bjorken- $x$. The typical $x$ value probed is $x \approx m_{W}^{2} / 2 m_{N} E_{\nu}$, which implies that for $E_{\nu} \approx 10^{8}-10^{10} \mathrm{GeV}$ one have $x \approx 10^{-4}-10^{-6}$ at $Q^{2} \approx 10^{4} \mathrm{GeV}^{2}$. This kinematical range was not explored by the HERA measurements of the structure functions 3 .

The description of QCD dynamics in such very high energy limit is a subject of intense debate 4 . Theoretically, at high energies (small Bjorken- $x$ ) one expects the transition of the regime described by the linear dynamics, where only the parton emissions are considered, to a new regime where the physical process of recombination of partons becomes important in the parton cascade and the evolution is given by a non-linear evolution equation. This regime is characterized by the limitation on the maximum phase-space parton density that can be reached in the hadron wavefunction (parton saturation), with the transition being specified by a 
typical scale, which is energy dependent and is called saturation scale $Q_{\mathrm{s}}$. Moreover, the growth of the parton distribution is expected to saturate, forming a Color Glass Condensate (CGC), whose evolution with energy is described by an infinite hierarchy of coupled equations for the correlators of Wilson lines 4 . In the mean field approximation, the first equation of this hierarchy decouples and boils down to a single non-linear integro-differential equation: the Balitsky-Kovchegov (BK) equation 516. Experimentally, possible signals of parton saturation have already been observed both in $e p$ deep inelastic scattering at HERA and in deuteron-gold collisions at RHIC 4 .

Currently, there are predictions of the neutrino nucleon cross sections with structure functions constrained by HERA data are based on linear dynamics 7 , using DGLAP or an unified DGLAP/BFKL evolution, or phenomenological models that resembles the expected behavior predicted by the non-linear QCD dynamics 8 (i.e., the proton structure function saturating the Froissart bound at asymptotic energies, $\left.F_{2}^{p} \propto \ln ^{2}(1 / x)\right)$. As a general feature, the nonlinear QCD dynamics predicts sizable suppression of UHE neutrino cross section in comparison with standard approaches. Here, we summarize the main results of works presented in Refs. 910 . In Ref. 9 the contribution of non-linear effects was estimated considering distinct phenomenological models based on saturation physics. An update on those calculations have been done recently 11 . In Ref. 10 , the geometric scaling property (which is a natural consequence of the asymptotic solutions of the nonlinear QCD evolution equations) is considered to obtain an analytical parameterization for the UHE neutrino cross sections. In what follows we introduce the theoretical and phenomenological tools and present the main results and predictions

\section{The UHE neutrino cross section and nonlinear perturbative QCD approaches}

Deep inelastic neutrino scattering is described in terms of charged current $(\mathrm{CC})$ and neutral current (NC) interactions, which proceed through $W^{ \pm}$and $Z^{0}$ exchanges, respectively. The total cross sections are given by:

$$
\begin{gathered}
\sigma_{\nu N}^{C C, N C}\left(E_{\nu}\right)=\int_{Q_{\min }^{2}}^{s} d Q^{2} \int_{Q^{2} / s}^{1} \frac{d x}{x s} \frac{G_{F}^{2} M E_{\nu}}{\pi}\left(\frac{M_{i}^{2}}{M_{i}^{2}+Q^{2}}\right)^{2} \\
\times\left[\frac{1+(1-y)^{2}}{2} F_{2}^{C C, N C}-\frac{y^{2}}{2} F_{L}^{C C, N C}+y\left(1-\frac{y}{2}\right) x F_{3}^{C C, N C}\right],
\end{gathered}
$$

where $E_{\nu}$ is the neutrino energy, $s=2 M E_{\nu}$ with $M$ the nucleon mass, $y=Q^{2} /(x s)$ and $Q_{\min }^{2}$ is the minimum value of $Q^{2}$ which is introduced in order to stay in the deep inelastic region. In what follows we assume $Q_{m i n}^{2}=1 \mathrm{GeV}^{2}$. Moreover, $G_{F}$ is the Fermi constant and $M_{i}$ denotes the mass of the charged of neutral gauge boson.

In the QCD improved parton model the structure functions $F_{i}\left(x, Q^{2}\right)$ are expressed in terms of the parton distributions on the nucleon, which satisfy the DGLAP 12 linear dynamics. On the other hand, an efficient way of introducing 
non-linear effects is the description of the structure functions considering the color dipole approach in which the DIS to low $x$ can be viewed as a result of the interaction of a color $q \bar{q}$ dipole which the gauge boson fluctuates 13 . In this approach the $F_{2}^{C C, N C}$ structure function is expressed in terms of the transverse and longitudinal structure functions, $F_{2}^{C C, N C}=F_{T}^{C C, N C}+F_{L}^{C C, N C}$ which are given by

$$
F_{T, L}^{C C, N C}\left(x, Q^{2}\right)=\frac{Q^{2}}{4 \pi^{2}} \int_{0}^{1} d z \int d^{2} r\left|\Psi_{T, L}^{W, Z}\left(r, z, Q^{2}\right)\right|^{2} \sigma^{d i p}(r, x)
$$

where $r$ denotes the transverse size of the dipole, $z$ is the longitudinal momentum fraction carried by a quark and $\Psi_{T, L}^{W, Z}$ are proportional to the wave functions of the virtual charged of neutral gauge bosons corresponding to their transverse or longitudinal polarizations. Explicit expressions for $\Psi_{T, L}^{W, Z}$ are given, e.g., in Ref. 9 . Furthermore, $\sigma^{\text {dip }}$ describes the interaction of the color dipole with the target. In next section we will discuss some models for $\sigma^{d i p}$, based on the non-linear QCD dynamics, which describe the current HERA data. As a comment, the DESY-HERA measurements of the structure function at low $-x\left(x \approx 10^{-6}\right)$ are for very low values of $Q^{2}\left(Q^{2} \ll 1 \mathrm{GeV}^{2}\right)$, which implies that small $x$ extrapolations of the parton distributions are necessary to estimate $\sigma_{\nu N}$ above $E_{\nu} \approx 10^{7} \mathrm{GeV}$.

As mentioned above, UHE neutrino-nucleon cross section accesses very large values of $Q^{2}$ and very small values of Bjorken $x$. The usual linear perturbative QCD evolution equations predictions are based on the linear DGLAP and/or BFKL equations which implies a power increase with the energy of the neutrino-nucleon cross section that eventually violate the Froissart bound. A easy way to introduce consistently the unitarity constraints on the theoretical estimate of the ultrahigh energy behavior of the neutrino-nucleon cross section is to express the structure functions in the dipole approach [Eq. (2)] and to consider the state-of-art of the nonlinear QCD dynamics: the Color Glass Condensate formalism. In this formalism, the dipole - target cross section $\sigma^{d i p}$ can be computed in the eikonal approximation, resulting

$$
\sigma^{d i p}(x, r)=2 \int d^{2} b \mathcal{N}(x, r, b)
$$

where $\mathcal{N}$ is the dipole-target forward scattering amplitude for a given impact parameter $b$ which encodes all the information about the hadronic scattering, and thus about the non-linear and quantum effects in the hadron wave function. It is useful to assume that the impact parameter dependence of $\mathcal{N}$ can be factorized as $\mathcal{N}(x, r, b)=\mathcal{N}(x, r) S(b)$, so that $\sigma^{d i p}(x, r)=\sigma_{0} \mathcal{N}(x, r)$, with $\sigma_{0}$ being a free parameter related to the non-perturbative QCD physics. The Balitsky-JIMWLK hierarchy describes the energy evolution of the dipole-target scattering amplitude $\mathcal{N}(x, r)$. In the mean field approximation, the first equation of this hierarchy decouples and boils down to the Balitsky-Kovchegov (BK) equation 516. The dipole-target cross section can also be calculated considering phenomenological parameterizations for $\mathcal{N}(x, r)$ based on saturation physics, which provide an economical description of a wide range of data with a few parameters. This approach has been considered in 
Refs. 9|11. In general, the dipole scattering amplitude is modeled in the coordinate space in terms of a simple Glauber-like formula as follows

$$
\mathcal{N}(x, r)=1-\exp \left[-\frac{1}{4}\left(r^{2} Q_{s}^{2}\right)^{\gamma\left(x, r^{2}\right)}\right],
$$

where $\gamma$ is the anomalous dimension of the target gluon distribution. The main difference among the distinct phenomenological models comes from the predicted behavior for the anomalous dimension, which determines the transition from the non-linear to the extended geometric scaling regimes, as well as from the extended geometric scaling to the DGLAP regime 4 .

One important property of the nonlinear perturbative QCD approaches for high energy deep inelastic $e p(A)$ scattering is the prediction of the geometric scaling. Namely, the total $\gamma^{*} p(A)$ cross section at large energies is not a function of the two independent variables $x$ and $Q^{2}$, but is rather a function of the single variable $\tau_{A}=Q^{2} / Q_{\text {sat,A. }}^{2}$. As usual, $Q^{2}$ is the photon virtuality and $x$ the Bjorken variable. Geometric scaling is the exact asymptotic solution of a general class of nonlinear evolution equations and it appears as a universal property of these kind of equations. The saturation momentum $Q_{\text {sat,A }}^{2}(x ; A) \propto \frac{x G_{A}\left(x, Q_{\mathrm{sat}}^{2}\right)}{\pi R_{A}^{2}} \simeq A^{\alpha} x^{-\lambda}(\alpha \simeq 1 / 3, \lambda \simeq$ $0.3)$ is connected with the phenomenon of gluon saturation. In principle, geometric scaling is predicted to be present only on process dominated by low momenta. However, it is known that the geometric scaling is preserved by the QCD evolution up to relatively large virtualities 4 , within the kinematical window $Q_{\text {sat }}^{2}(x)<Q^{2}<$ $Q_{\mathrm{sat}}^{4}(x) / \Lambda_{\mathrm{QCD}}^{2}$. That is, the scaling property extends towards very large virtualities provided one stays in low- $x$. This kinematical window is further enlarged due to the nuclear enhancement of the saturation scale. These facts have direct consequences in the behavior of UHE neutrino cross section.

As similar scaling holds for the massive boson-nucleon cross section, we are able to compute analytically the neutrino cross sections. This feature was verified to be true in Ref. 14, where geometric scaling was shown to occur in the small- $x$ charged current neutrino data. In this sense, we are able to construct a theoretical prediction which is model independent. They are as follows 10 ,

$$
\sigma_{(\nu, \bar{\nu})}^{\mathrm{CC}, \mathrm{NC}}=\mathcal{N}_{(i)} A^{\alpha}\left(\frac{R_{A}^{2}}{R_{p}^{2}}\right)^{1-\alpha}\left[C_{1}^{(i)} E_{\nu}^{\omega_{\text {scal }}}-C_{2}^{(i)}\right],
$$

where $\mathcal{N}_{(i)}$ are overall normalizations, $C_{1,2}^{(i)}$ are numerical constants with $i=$ CC, $\mathrm{NC}, \omega_{\text {scal }}=b \lambda$ and $\alpha=b / \delta$. This implies in a mild power-like rise $\omega_{\text {scal }} \simeq 0.2$ for the neutrino cross section in contrast with other theoretical approaches. The nuclear dependence is approximately linear, $\sigma_{\nu, \bar{\nu}}^{\text {nuclei }} \propto A \sigma_{\nu, \bar{\nu}}^{\text {nucleon }}$, once $b \simeq \delta$ and hence $\alpha \approx 1$. The remaining constants are given by (see Ref. 10 for details),

$$
\begin{aligned}
& \mathcal{N}_{(i)}=R_{\text {cor }}\left(\frac{\bar{\sigma}_{0} G_{F}^{2} M_{W, Z}^{2}}{8 \pi^{3} \lambda}\right)\left(\frac{a x_{0}^{\omega_{\text {scal }}}}{b}\right) \frac{B_{(i)}}{\alpha_{\mathrm{em}} \sum_{f} e_{f}^{2}}, \\
& C_{1}^{(i)}=\pi\left(2 m_{N}\right)^{\omega_{\text {scal }}}\left(M_{W, Z}^{2}\right)^{-\nu_{\text {scal }}} \csc \left(\pi \nu_{\text {scal }}\right)\left(1-\nu_{\text {scal }}\right), C_{2}^{(i)}=\pi\left(M_{W, Z}^{2}\right)^{-b} \csc (\pi b)(1-b),
\end{aligned}
$$


September $\quad 28, \quad 2018 \quad 13: 45 \quad$ WSPC/INSTRUCTION $\quad$ FILE proc magno machado stars2011

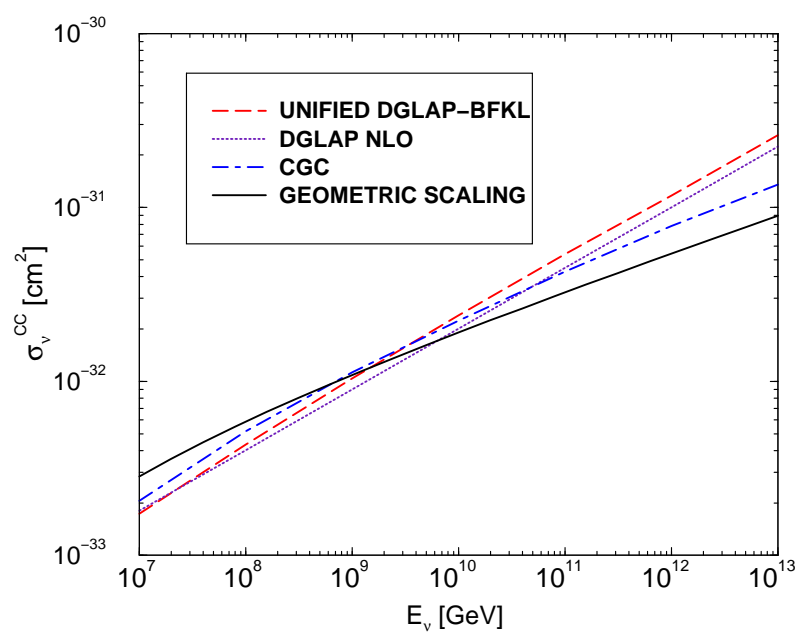

Fig. 1. Comparison among perturbative QCD approaches: unified DGLAP-BFKL, NLO DGLAP and a phenomenological model based on the Color Glass Condensate physics (CGC). The analytical geometric scaling parameterization is also presented.

where one uses the notation $B_{\mathrm{CC}}=4, B_{\mathrm{NC}}=K_{\text {chiral }}$ and $\nu_{\text {scal }}=b-\omega_{\text {scal }}$. Numerically, this gives a total cross section $\sigma_{\nu, \bar{\nu}}^{t o t}=1.48 \times 10^{-34} A^{\alpha}\left(E_{\nu} / \mathrm{GeV}\right)^{0.227} \mathrm{~cm}^{2}$. The cross section above can have implications for neutrino observatories because experiments are planned to detect UHE by observation of the nearly horizontal air showers in Earth coming from neutrino-air interactions 15 . A reduced cross section produces a smaller event rate for such neutrino-induced showers and could compromise the detection signal. However, the rate of up-going air showers initiated by muon and tau leptons produced in neutrino-nucleon reactions just below the surface would increase, being possibly larger than the horizontal air shower rate.

\section{Results and summary}

Let us present the comparison between the predictions of the linear approaches (NLO DGLAP and unified DGLAP/BFKL) and non-linear QCD approaches (a phenomenological model for the Color Glass Condensate and the geometric scaling prediction). In Fig. 1 the energy dependence of the neutrino nucleon $\mathrm{CC}$ cross section predicted by the linear and nonlinear perturbative QCD approaches are compared. As expected from the solution of the DGLAP equation at small- $x$, the NLO DGLAP (dot-dashed curve) and unified DGLAP/BFKL (long-dashed curve) results predict a strong increase of the cross section at ultrahigh energies. Such estimates are largely used to estimate the event rates in neutrinos telescopes. Clearly, the CGC calculation predicts an increasingly suppressed cross section compared to its linear QCD counterpart.

In Fig. 1 the solid line represents the result of geometric scaling parameteri- 
September $28, \quad 2018 \quad 13: 45$ WSPC/INSTRUCTION $\quad$ FILE proc magno machado 'stars2011

M. V. T. Machado

zation, Eq. (5). The suppression in the cross section is even higher than for the CGC phenomenological models reaching a factor two compared the the linear QCD approaches. The present results demonstrate that the determination of $\sigma_{\nu N}$ can be useful to constrain the underlying QCD dynamics. In principle, this cross section could be constrained at high energies by studying the ratio between quasi-horizontal deeply penetrating air showers and Earth-skimming tau showers 15 .

As a summary, the detection of UHE neutrinos may shed light on the observation of air showers events with energies in excess of $10^{11} \mathrm{GeV}$, reveal aspects of new physics as well as of the QCD dynamics at high energies. One of the main ingredients for estimating event rates in neutrino telescopes and cosmic ray observatories (e.g. AUGER) is the neutrino - nucleon cross section. We have examined to what extent the cross section is sensitive to the presence of new dynamical effects in the QCD evolution. We compare the predictions of several approaches based on different assumptions for the QCD dynamics. In particular, we have compared the phenomenological models based on nonlinear perturbative QCD with the usual NLO DGLAP evolution. Moreover, we compute a model independent cross section, using the experimental verification of the geometric scaling property. The results demonstrate that the current theoretical uncertainty for the neutrino-nucleon cross section reaches a factor two or higher for neutrino energies above $10^{11} \mathrm{GeV}$.

\section{References}

1. L. A. Anchordoqui and T. Montaruli, Ann. Rev. Nucl. Part. Sci. 60 (2010) 129.

2. S. Andriga [Pierre Auger Collaboration], arXiv.1005.3795 [hep-ex].

3. G. Wolf, Rept. Prog. Phys. 73 (2010) 116202.

4. F. Gelis, E. Iancu, J. Jalilian-Marian and R. Venugopalan, Ann. Rev. Nucl. Part. Sci. 60 (2010) 463.

5. I. I. Balitsky, Nucl. Phys. B463 (1996) 99; Phys. Rev. Lett. 81 (1998) 2024.

6. Y.V. Kovchegov, Phys. Rev. D 60 (1999) 034008; Phys. Rev. D 61 (2000) 074018.

7. A. Cooper-Sarkar and S. Sarkar, JHEP 0801 (2008) 075.

8. M. M. Block, P. Ha and D. W. McKay, Phys. Rev. D 82 (2010) 077302.

9. M. V. T. Machado, Phys. Rev. D 70 (2004) 053008.

10. M. V. T. Machado, Phys. Rev. D 71 (2005) 114009.

11. V. P. Goncalves, P. Hepp, Phys. Rev. D 83 (2011) 014014.

12. V.N. Gribov and L.N. Lipatov, Sov. J. Nucl. Phys. 15 (1972) 438; G. Altarelli and G. Parisi, Nucl. Phys. B126 (1977) 298; Yu.L. Dokshitzer, Sov. Phys. JETP 46 (1977) 641.

13. N. N. Nikolaev, B. G. Zakharov, Phys. Lett. B 332 (1994) 184; Z. Phys. C 64 (1994) 631.

14. M. B. Gay Ducati, M. M. Machado and M. V. T. Machado, Phys. Lett. B, 644b (2007) 340 .

15. L. A. Anchordoqui, H. Goldberg, D. Gora, T. Paul, M. Roth, S. Sarkar and L. L. Winders, Phys. Rev. D 82 (2010) 043001. 